\title{
$\beta$-decay rates of ${ }^{121-131} \mathrm{Cs}$ in the microscopic interacting boson-fermion model
}

\author{
E. Mardones and J. Barea* \\ Departamento de Física, Universidad de Concepción, Casilla 160-C, Concepción, 4070386 Chile \\ C. E. Alonso and J. M. Arias \\ Departamento de Física Atómica, Molecular y Nuclear, Universidad de Sevilla, Apartado 1065, 41080 Sevilla, Spain
}

(Received 17 December 2015; published 25 March 2016)

\begin{abstract}
$\beta$-decay rates of ${ }^{121-131} \mathrm{Cs}$ have been calculated in the framework of the neutron-proton interacting bosonfermion model (IBFM-2). For odd- $A$ nuclei, the decay operator can be written in a relatively simple form in terms of the one-nucleon transfer operator. Previous studies of $\beta$ decay in IBFM-2 were based on a transfer operator obtained by using the number operator approximation (NOA). In this work a new form of the one-nucleon transfer operator, derived microscopically without the NOA approximation, is used. The results from both approaches are compared and show that the deviation from experimental data is reduced without using the NOA approximation. Indications about the renormalization of the Fermi and Gamow-Teller matrix elements are discussed. This is a further step toward a more complete description of low-lying states in medium and heavy nuclei which is necessary to compute reliable matrix elements in studies of current active interest such as double- $\beta$ decay or neutrino absorption experiments.
\end{abstract}

DOI: 10.1103/PhysRevC.93.034332

\section{INTRODUCTION}

Weak interaction is present in many particle physics processes, some of them with a high impact in nuclear astrophysics. Even when the Standard Model describes this fundamental interaction reasonably well, there are still some unanswered questions related to it as, for instance, the nature of the neutrino and its mass. Weak interaction affects all leptons and quarks, in contrast to the strong interaction, which only affects quarks, or the electromagnetic interaction which only affects charged particles. Thus, the universal character of this interaction makes relevant its study. In nuclear physics, $\beta$ decay is the most common manifestation of the weak interaction and is extremely significant because it is one of the main mechanisms that atomic nuclei have to reach stability. Alternatively there are two processes related to $\beta$ decay which still require further investigation. The first one is related to the gallium neutrino source experiments, where the ratio between the measured and calculated ${ }^{71} \mathrm{Ga}$ cross sections for absorbing

${ }^{51} \mathrm{Cr}$ neutrinos is smaller than unity, more widely known as the gallium anomaly. The second one is the double- $\beta$ decay. Here both the neutrinoless and two-neutrino modes are currently of great interest for particle and nuclear physics. Lately an active discussion arose on a long-standing problem in nuclear physics, which is the effective value of the axial coupling constant $g_{A}$. It is important then to rely on a nuclear structure model that is able to provide a unified framework to compute the necessary matrix elements involved in all these processes.

The interacting boson model (IBM) [1] has demonstrated thoroughly its capacity to describe spectra, electromagnetic transitions and moments and other properties of the low-lying collective states in even-even medium and heavy nuclei. By coupling a fermion to the system of bosons, which describes the states of even-even nuclei, the low-lying states of adjacent odd- $A$ nuclei can be obtained. This extension of the model is

\footnotetext{
*jbarea@udec.cl
}

called the interacting boson-fermion model (IBFM) [2]. The IBM can be formulated without distinguishing neutron and proton bosons (IBM-1) [3] or introducing the neutron-proton degree of freedom (IBM-2) [4]. Accordingly the IBFM-1 [5] does not distinguish between neutrons and protons, while IBFM-2 [6] does, which makes it suitable to compute $\beta$-decay rates between odd-even nuclei. This decay is modeled in the IBM framework as the combination of a neutron (proton) stripping and proton (neutron) pickup reactions for the $\beta^{-}$ $\left(\beta^{+}\right.$, electron capture) decay. Then, the explicit form of the one-nucleon transfer operator [7] is needed. Such an operator was originally derived within the generalized seniority (GS) scheme [8] using the number operator approximation (NOA) [9] by obtaining the image of the shell-model one nucleon (proton or neutron) creation operator in the IBFM-2 space, following the Otsuka, Arima \& Iachello (OAI) method [10]. The first proposal to study $\beta$-decay rates in this scheme was done by Dellagiacoma and Iachello [11]. Later on similar studies were presented by Zuffi et al.[12] using the same formalism and transfer operator. In these last studies, the parameters in the boson-fermion Hamiltonian (single-particle energies for the odd fermion and boson-fermion interaction parameters) were fitted for each nucleus independently so as to reproduce its spectroscopic properties and $\beta$-decay rates.

Very recently the IBM one-nucleon transfer operator has been microscopically rederived by suppressing the use of the NOA approximation [13]. The new operator was originally checked by calculating one-nucleon transfer intensities. A more demanding test to the new operator is to use it for calculating $\beta$-decay transition rates. In this work such a study is presented. In contrast to the work of Zuffi and collaborators, our aim is not to give the best individual description of each nucleus but to show whether the effects of removing the NOA approximation in the calculation of $\beta$-decay rates improves the results. Thus, the study is performed without any parameter fitting, all parameters for even-even core nuclei, single-particle energies for fermions, and boson-fermion interaction were 
taken from a previous systematic work [14]. In this spirit, a calculation of $\beta$-decay rates of ${ }^{121-131} \mathrm{Cs}$ is presented here using the new transfer operator. The results are compared against those obtained using the NOA approximation (under the same conditions) and the experimental values. Finally estimations of the renormalizations of the matrix elements with respect to those calculated using the free-nucleon single-particle matrix elements are performed.

The article is structured as follows. In Sec. II, the formalism for $\beta$ decay in IBM is briefly reviewed. In Sec. III, the calculated $\log f t$ values are presented and compared with those obtained using the NOA approximation and the experimental ones. Finally, in Sec. IV, the conclusions of this work are briefly discussed.

\section{FORMALISM}

We will not enter into details of the IBFM-2 model which can be found elsewhere $[2,6,14]$. Instead we concentrate on the description of the $\beta$-decay operator and on how the $f t$ values are calculated. For this purpose we follow the formalism developed in Ref. [11].

We use the following expression to obtain the $f t$ values (in seconds):

$$
f t=\frac{6163}{M_{\mathrm{F}}^{2}+\left(\frac{g_{A}}{g_{V}}\right)^{2} M_{\mathrm{GT}}^{2}} \mathrm{~s},
$$

where the ratio $g_{A} / g_{V}=-1.2756(30)$ [15] is adopted and $M_{\mathrm{F}}$ and $M_{\mathrm{GT}}$ are the matrix elements of the Fermi and GamowTeller operators, $T_{\mathrm{F}}$ and $T_{\mathrm{GT}}$, respectively, between the states of the parent and daughter nuclei. These operators can be written for the $\beta^{-}$decay in terms of the transfer operators $c_{\rho, i}^{\dagger}, \tilde{c}_{\rho, i}$ ( $\rho=v$ for neutrons and $\rho=\pi$ for protons) as

$$
\begin{aligned}
T_{\mathrm{F}} & =-\sum_{i} \hat{j}_{i}\left[A_{\pi, i} c_{\pi, i}^{\dagger} \times A_{v, i} \tilde{c}_{v, i}\right]^{(0)}, \\
T_{\mathrm{GT}, \mu} & =\sum_{i, i^{\prime}} \sigma_{i i^{\prime}}\left[A_{\pi, i} c_{\pi, i}^{\dagger} \times A_{\nu, i^{\prime}} \tilde{c}_{v, i^{\prime}}\right]_{\mu}^{(1)},
\end{aligned}
$$

where the index $i$ denotes a particular shell, specified by the standard single-particle level quantum numbers $n_{i}, l_{i}, \frac{1}{2}, j_{i}$, and $m_{i}$, and $\hat{j}_{i}=\sqrt{2 j_{i}+1}$. The $\beta^{+}$and electron-capture decay operators can be obtained by exchanging $\pi$ and $\nu$. The above sums run over the single-particle orbits included in the model space and $\sigma_{i i^{\prime}}$ is given by

$$
\begin{aligned}
\sigma_{i i^{\prime}} & =-\frac{1}{\sqrt{3}}\left\langle l_{i} \frac{1}{2} ; j_{i}\|\vec{\sigma}\| l_{i^{\prime}} \frac{1}{2} ; j_{i^{\prime}}\right) \delta_{l_{i} l_{i^{\prime}}} \\
& =(-1)^{l_{i}+j_{i}-3 / 2} \hat{j}_{i} \hat{j}_{i^{\prime}} \sqrt{2}\left\{\begin{array}{ccc}
\frac{1}{2} & \frac{1}{2} & 1 \\
j_{i^{\prime}} & j_{i} & l
\end{array}\right\} \delta_{l_{i} l_{i^{\prime}}},
\end{aligned}
$$

where $\vec{s}=\vec{\sigma} / 2$ is the spin operator. The transfer operators $c_{\rho, i}^{\dagger}$ read

$$
\begin{aligned}
c_{\rho, i}^{\dagger}= & \frac{\eta_{\rho, 2 N_{\rho}, 1, j_{i}}}{\eta_{\rho, 2 N, 0,0}} a_{\rho, i}^{\dagger}+\sqrt{N_{\rho}} \alpha_{\rho, i} \frac{\eta_{\rho, 2\left(N_{\rho}-1\right), 1, j_{i}}}{\eta_{\rho, 2 N_{\rho}, 0,0}} \\
& \times\left(s_{\rho}^{\dagger} \times \tilde{a}_{\rho, i}\right)_{m_{i}}^{\left(j_{i}\right)}+\sum_{i^{\prime}=1}^{k} \frac{\sqrt{5} \beta_{\rho, i^{\prime} i}}{\hat{j}_{i} \sqrt{1+\delta_{i i^{\prime}}}}
\end{aligned}
$$

$$
\begin{aligned}
& \times \frac{\eta_{\rho, 2 N_{\rho}, 2,2}^{2}\left(i i^{\prime}\right)}{\eta_{\rho, 2 N_{\rho}, 2,2} \eta_{\rho, 2\left(N_{\rho}-1\right), 1, j_{i^{\prime}}}}\left(d_{\rho}^{\dagger} \times \tilde{a}_{\rho, i^{\prime}}\right)_{m_{i}}^{\left(j_{i}\right)} \\
& -\sum_{i^{\prime}=1}^{k} \frac{\sqrt{5 N_{\rho}} \alpha_{\rho, i} \beta_{\rho, i^{\prime} i} \sqrt{1+\delta_{i i^{\prime}}}}{\hat{j_{i}}} \\
& \times \frac{\eta_{\rho, 2 N_{\rho}, 2,2}^{2}\left(i i^{\prime}\right)}{\eta_{\rho, 2 N_{\rho}, 2,2} \eta_{\rho, 2 N_{\rho}, 1, j_{i^{\prime}}}} s_{\rho}^{\dagger}\left(\tilde{d}_{\rho} \times a_{\rho, i^{\prime}}^{\dagger}\right)_{m_{i}}^{\left(j_{j}\right)},
\end{aligned}
$$

where the $\eta_{\rho, n_{\rho}, \tilde{\mathrm{v}} J}, \alpha_{\rho, i}$, and $\beta_{\rho, i^{\prime} i}$ quantities are defined in [13] and $N_{\rho}$ is the number of bosons of type $\rho$. We give for completeness the expression of the transfer operator obtained under the NOA approximation

$$
\begin{aligned}
c_{\rho, i}^{\dagger}= & \sqrt{1-\alpha_{\rho, i}^{2} \frac{N_{\rho}}{\Omega_{\rho, e}}} a_{\rho, i}^{\dagger}+\frac{\alpha_{\rho, i}}{\sqrt{\Omega_{\rho, e}}}\left(s_{\rho}^{\dagger} \times \tilde{a}_{\rho, i}\right)_{m_{i}}^{\left(j_{i}\right)} \\
& +\sum_{i^{\prime}=1}^{k} \sqrt{1-\alpha_{\rho, i}^{2} \frac{N_{\rho}}{\Omega_{\rho, e}}} \frac{\sqrt{10} \beta_{\rho, i^{\prime} i}}{\hat{j}_{i} K_{\beta, \rho}}\left(d_{\rho}^{\dagger} \times \tilde{a}_{\rho, i^{\prime}}\right)_{m_{i}}^{\left(j_{i}\right)} \\
& -\sum_{i^{\prime}=1}^{k} \frac{\alpha_{\rho, i}}{\sqrt{\Omega_{\rho, e}}} \frac{\sqrt{10} \beta_{\rho, i^{\prime} i}}{\hat{j}_{i} K_{\beta, \rho}} s_{\rho}^{\dagger}\left(\tilde{d}_{\rho} \times a_{\rho, i^{\prime}}^{\dagger}\right)_{m_{i}}^{\left(j_{i}\right)},
\end{aligned}
$$

where $\Omega_{\rho, e}=\sum_{i} \alpha_{\rho, i}^{2} \hat{j}_{i}^{2} / 2$ and $K_{\beta, \rho}=\sqrt{\sum_{i i^{\prime}} \beta_{\rho, i^{\prime} i}^{2}}$.

The coefficients $A_{\rho, i}$ in Eqs. (2) and (3) allow us to fix a specific normalization for the transfer operators. In this work they were fixed by imposing the Macfarlane sum rule [16]

$$
\sum_{\alpha_{o} J_{o}}\left\langle\alpha_{o} J_{o}\left\|A_{\rho, i} c_{\rho, i}^{\dagger}\right\| \alpha_{e} J_{e}\right\rangle^{2}=\left(2 j_{i}+1\right) u_{\rho, i}^{2},
$$

when the even-even nucleus is the core of the odd-even nucleus, whose states are denoted by $\alpha_{e} J_{e}$ and $\alpha_{o} J_{o}$ respectively. Otherwise the following Macfarlane sum rule is used:

$$
\sum_{\alpha_{o} J_{o}}\left\langle\alpha_{e} J_{e}\left\|A_{\rho, i} c_{\rho, i}^{\dagger}\right\| \alpha_{o} J_{o}\right\rangle^{2}=\left(2 j_{i}+1\right) v_{\rho, i}^{2},
$$

where $u_{i}$ and $v_{i}$ are the occupation probabilities obtained from a BCS calculation. These quantities were used also to fix the values of $\alpha_{\rho, i}$ and $\beta_{\rho, i^{\prime} i}$ as

$$
\begin{aligned}
\alpha_{\rho, i} & =v_{\rho, i} \sqrt{\frac{\Omega_{\rho, e}}{N_{\rho}}}, \\
\beta_{\rho, i i^{\prime}} & =\left(u_{\rho, i} v_{\rho, i^{\prime}}+u_{\rho, i^{\prime}} v_{\rho, i}\right) Q_{i i^{\prime}},
\end{aligned}
$$

where $Q_{i i^{\prime}}$ are the single-particle matrix elements of the quadrupole operator.

To evaluate the matrix elements $M_{\mathrm{F}}$ and $M_{\mathrm{GT}}$ we developed FORTRAN codes which make use of standard reduction formulas derived for tensor operators [17]

$$
\begin{aligned}
M_{\mathrm{F}}= & \left\langle D ; J_{D}\left\|T_{\mathrm{F}}\right\| P ; J_{P}\right\rangle / \sqrt{2 J_{P}+1} \\
= & \frac{1}{2 J_{P}+1} \sum_{i, I}(-1)^{J_{P}+j_{i}+J_{I}} \delta_{J_{P} J_{D}} \\
& \times\left\langle D ; J_{D}\left\|A_{\pi, i} c_{\pi, i}^{\dagger}\right\| I ; J_{I}\right\rangle\left\langle I ; J_{I}\left\|A_{v, i} \tilde{c}_{v, i}\right\| P ; J_{P}\right\rangle,
\end{aligned}
$$



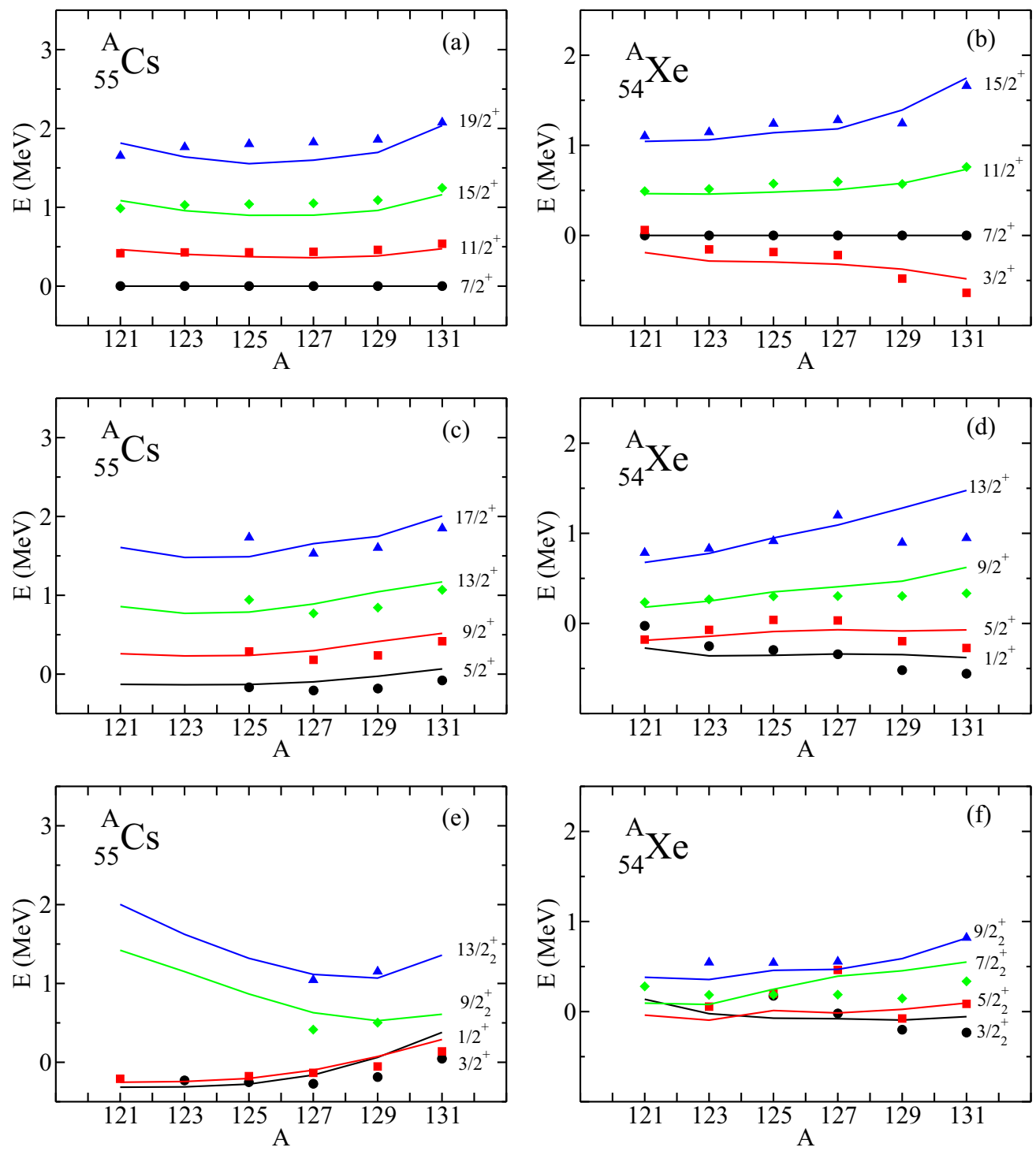

FIG. 1. Excitation spectra of Cs and Xe isotopes for positive parity. The energy of the first $\frac{7}{2}^{+}$state was taken as the zero of the energy. Lines correspond to calculated energies, and experimental data (symbols) were extracted from [18].

$$
\begin{aligned}
M_{\mathrm{GT}}= & \left\langle D ; J_{D}\left\|T_{\mathrm{GT}}\right\| P ; J_{P}\right\rangle / \sqrt{2 J_{P}+1} \\
= & \sum_{i i^{\prime} I}(-1)^{J_{P}+J_{D}+l_{i}+j_{i}+3 / 2} \sqrt{6} \frac{\hat{j_{i}} \hat{j}_{i^{\prime}}}{\hat{J}_{P}} \\
& \times\left\{\begin{array}{ccc}
\frac{1}{2} & \frac{1}{2} & 1 \\
j_{i^{\prime}} & j_{i} & l
\end{array}\right\}\left\{\begin{array}{lll}
j_{i} & j_{i^{\prime}} & 1 \\
J_{P} & J_{D} & J_{I}
\end{array}\right\} \delta_{l_{i} l_{i^{\prime}}} \\
& \times\left\langle D ; J_{D}\left\|A_{\pi, i} c_{\pi, i}^{\dagger}\right\| I ; J_{I}\right\rangle\left\langle I ; J_{I}\left\|A_{v, i^{\prime}} \tilde{c}_{v, i^{\prime}}\right\| P ; J_{P}\right\rangle,
\end{aligned}
$$

where $P, D$, and $I$ stand for parent, daughter, and intermediate nuclei. The intermediate nucleus is the even-even one adjacent to the odd-even nuclei involved. It is understood that parity is conserved, since we are working on allowed transitions.

\section{RESULTS AND DISCUSSION}

We have applied the above formalism to the isotopic chain ${ }^{121-131}$ Cs. This chain was studied in [14], the excitation spectra and some electromagnetic properties experimentally measured at that time were successfully reproduced. We have taken from [14] the parameters for the even-even core nuclei, the single-particle energies, and the boson-fermion interaction parameters. It is worth noting that in those calculations there was a truncation in the number of core states, retaining only those below $3 \mathrm{MeV}$. In the present study we have followed the same prescription to keep the same values for the boson-fermion interaction parameters. In Fig. 1 the calculated excitation energies are shown along with the updated experimental values for the Cs and Xe isotopes studied. We can see that the present experimental picture that includes new data is well reproduced by the old systematic calculation confirming the predictive 
TABLE I. Log $f t$ values of the $\beta$ decay of ${ }^{121-131}$ Cs for the "Truncated" calculations.

\begin{tabular}{|c|c|c|c|c|}
\hline \multirow[t]{2}{*}{$\beta^{+}$transition } & \multirow[t]{2}{*}{$J_{\text {g.s. }} \rightarrow J_{f}$} & \multicolumn{3}{|c|}{$\log f t$} \\
\hline & & $\begin{array}{l}\text { With } \\
\text { NOA }\end{array}$ & $\begin{array}{l}\text { Without } \\
\text { NOA }\end{array}$ & Expt. \\
\hline${ }^{121} \mathrm{Cs} \rightarrow{ }^{121} \mathrm{Xe}$ & $\begin{array}{l}3 / 2_{1} \rightarrow 5 / 2_{1} \\
3 / 2_{1} \rightarrow 1 / 2_{1} \\
3 / 2_{1} \rightarrow 3 / 2_{1}\end{array}$ & $\begin{array}{l}6.152 \\
6.654 \\
5.646\end{array}$ & $\begin{array}{l}6.124 \\
6.608 \\
4.570\end{array}$ & $\begin{array}{l}5.5(3) \\
6.1(3) \\
6.2(3)\end{array}$ \\
\hline${ }^{123} \mathrm{Cs} \rightarrow{ }^{123} \mathrm{Xe}$ & $\begin{array}{l}1 / 2_{1} \rightarrow 1 / 2_{1} \\
1 / 2_{1} \rightarrow 3 / 2_{1}\end{array}$ & $\begin{array}{l}5.894 \\
6.059\end{array}$ & $\begin{array}{l}4.673 \\
5.979\end{array}$ & $\begin{array}{l}5.62(13) \\
5.65(6)\end{array}$ \\
\hline${ }^{125} \mathrm{Cs} \rightarrow{ }^{125} \mathrm{Xe}$ & $\begin{array}{l}1 / 2_{1} \rightarrow 1 / 2_{1} \\
1 / 2_{1} \rightarrow 3 / 2_{1} \\
1 / 2_{1} \rightarrow 1 / 2_{1} \\
1 / 2_{1} \rightarrow 1 / 2_{2}\end{array}$ & $\begin{array}{l}6.506 \\
6.355 \\
5.004 \\
5.741\end{array}$ & $\begin{array}{l}5.116 \\
6.216 \\
5.524 \\
4.867\end{array}$ & $\begin{aligned} \approx & 5.6 \\
\approx & 6.76 \\
& 6.53(6) \\
& 5.558(11)\end{aligned}$ \\
\hline${ }^{127} \mathrm{Cs} \rightarrow{ }^{127} \mathrm{Xe}$ & $\begin{array}{l}1 / 2_{1} \rightarrow 3 / 2_{1} \\
1 / 2_{1} \rightarrow 3 / 2_{2} \\
1 / 2_{1} \rightarrow 3 / 2_{3} \\
1 / 2_{1} \rightarrow 3 / 2_{4}\end{array}$ & $\begin{array}{l}8.526 \\
7.156 \\
7.459 \\
7.276\end{array}$ & $\begin{array}{l}7.961 \\
7.472 \\
8.017 \\
7.280\end{array}$ & $\begin{array}{l}6.791(24) \\
7.574(20) \\
8.83(10) \\
6.306(12)\end{array}$ \\
\hline${ }^{129} \mathrm{Cs} \rightarrow{ }^{129} \mathrm{Xe}$ & $\begin{array}{l}1 / 2_{1} \rightarrow 1 / 2_{1} \\
1 / 2_{1} \rightarrow 1 / 2_{2} \\
1 / 2_{1} \rightarrow 3 / 2_{1} \\
1 / 2_{1} \rightarrow 3 / 2_{2} \\
1 / 2_{1} \rightarrow 3 / 2_{3}\end{array}$ & $\begin{array}{l}4.900 \\
5.937 \\
8.989 \\
5.885 \\
6.263\end{array}$ & $\begin{array}{l}5.056 \\
5.314 \\
8.313 \\
5.837 \\
6.184\end{array}$ & $\begin{array}{l}6.24(6) \\
5.6(4) \\
7.28(20) \\
7.1(3) \\
6.4(5)\end{array}$ \\
\hline${ }_{\mathrm{rms}}^{131} \mathrm{Cs} \rightarrow{ }^{131} \mathrm{Xe}$ & $5 / 2_{1} \rightarrow 3 / 2_{1}$ & $\begin{array}{l}5.676 \\
0.945\end{array}$ & $\begin{array}{l}5.815 \\
0.843\end{array}$ & $5.548(20)$ \\
\hline
\end{tabular}

power of the model. This ensures that the boson-fermion parameters obtained in [14] are robust.

With the obtained wave functions, we have calculated the $\log f t$ values using the IBM one-nucleon transfer operator with and without the NOA approximation as shown in Table I. Our goal was to confirm if, under the same conditions, the new operator without NOA works better than the one that uses NOA. In addition, for each case we made two calculations. In one of them the states considered in the odd nucleus to impose the Macfarlane sum rules for the transfer operator were below $3 \mathrm{MeV}$ consistently with the even-even core calculation. The other one considers all the resulting states in the odd nucleus (no cutoff in energy). The Macfarlane sum rules, whose imposition fixes different normalization to the involved transfer operators, provides different values for the $\log f t$, even when the nuclear states are exactly the same in both types of calculations. To study the global goodness of each calculation, we computed the rms deviation from the experimental values presented in Table I. The "Truncated" calculation produces an rms value equal to 0.843 for the calculation without the NOA approximation, while the "Full" calculation (not shown in the table) brings the value 1.427. In the case of the "Truncated" calculation using the NOA approximation the rms value is slightly higher and equal to 0.945 . The conclusion was that a consistent calculation with the same cutoff energy in the even-even and odd-even systems is better than including as many states in the odd system as resulting from the coupling. We found that $3 \mathrm{MeV}$ is a reasonable choice to fix the cutoff in the odd-even system, just as it was done for the core states, because the single-particle states included in the calculation are in this range. The bottom line is that the
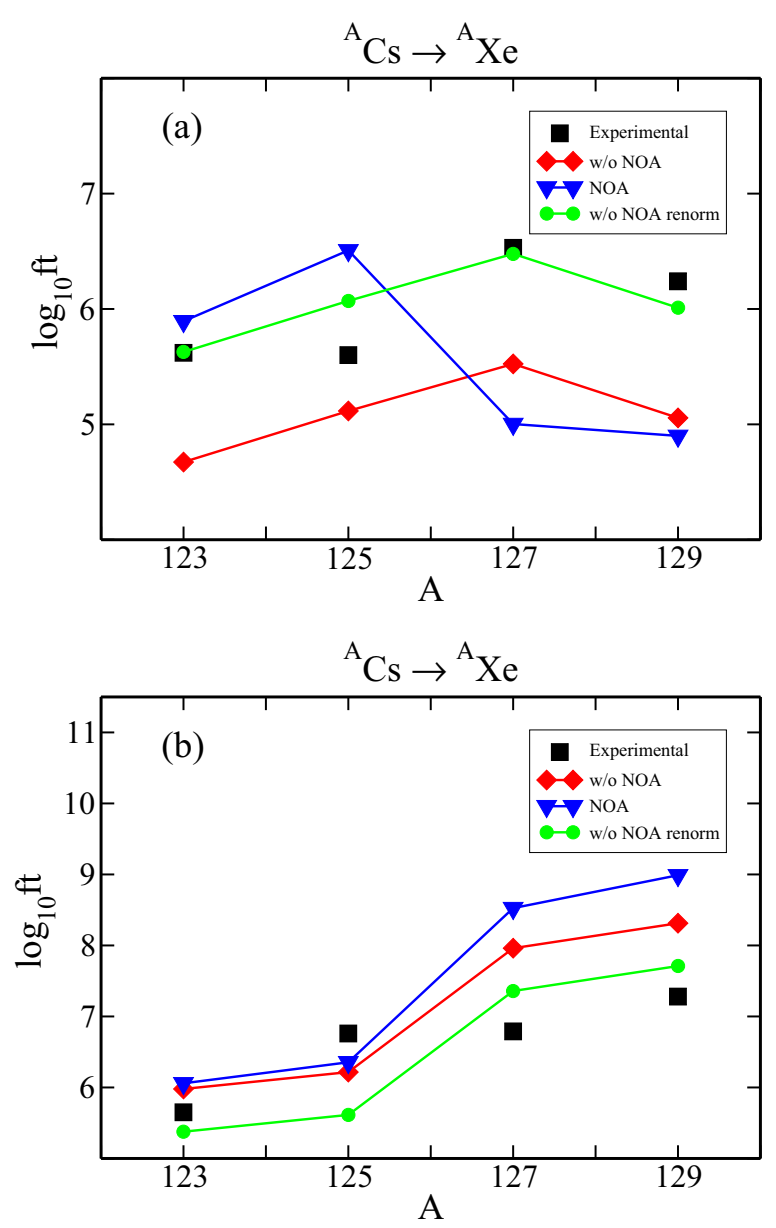

FIG. 2. Log $f t$ values from $1 / 2_{1}^{+}$states in Cs isotopes to (a) $1 / 2_{1}^{+}$ and (b) $3 / 2_{1}^{+}$states in Xe isotopes.

truncation scheme without the NOA approximation improves the overall agreement. All the results discussed below were obtained with the energy cutoff at $3 \mathrm{MeV}$.

In Table I we can observe that the values obtained with and without the NOA approximation are close in general. From the global fit, however, it is clear by looking at the rms that the calculations performed without NOA give better results than the old one obtained within the NOA approximation. When we compare the calculated $\log f t$ values with the experimental values, the major discrepancy can be found in the decay $3 / 2_{1}^{+} \rightarrow 3 / 2_{1}^{+}$in ${ }^{121} \mathrm{Cs} \rightarrow{ }^{121} \mathrm{Xe}$ for the calculation without the NOA approximation. When the NOA approximation is used, the main differences are found in the decays $1 / 2_{1}^{+} \rightarrow$ $3 / 2_{1}^{+}$in ${ }^{127} \mathrm{Cs} \rightarrow{ }^{127} \mathrm{Xe}$ and ${ }^{129} \mathrm{Cs} \rightarrow{ }^{129} \mathrm{Xe}$. The origin of these differences can be the absence of higher-lying single-particle orbitals for neutrons in the calculations. For instance the inclusion of the $1 h_{9 / 2}$ orbital would improve the quality of the calculated values, as was mentioned in [14] in the discussion about the $B(M 1)$ and magnetic moments.

In Fig. 2 we have plotted the calculated and experimental $\log f t$ values for the decay from the $1 / 2_{1}^{+}$states of Cs isotopes to the $1 / 2_{1}^{+}$and $3 / 2_{1}^{+}$states of Xe isotopes quoted in Table I with and without the NOA approximation. The structure of both the $\mathrm{Cs}$ and the $\mathrm{Xe}$ isotopes in the region studied in this 
work is rather complicated as shown in Fig. 1. Despite this complication, we can observe that the calculated values for the transitions $1 / 2_{1}^{+} \rightarrow 1 / 2_{1}^{+}$without the NOA approximation follow nicely the experimental trend. In contrast the values obtained with the NOA approximation show a rather erratic behavior when compared with the experimental points. The origin of this behavior is unclear and difficult to single out in such a complex and realistic calculation like this one, but the results support the removal of unnecessary approximations like NOA. In the case of the transitions $1 / 2_{1}^{+} \rightarrow 3 / 2_{1}^{+}$, both calculations follow the experimental trend, but again the calculation without NOA gets closer values to the experimental ones. These facts confirm the goodness of the calculations without NOA, what encourages us to obtain the complete boson-fermion interaction without this approximation.

In addition, some effects coming from higher-order corrections to the $\beta$-decay operator may also be relevant, as discussed in [19-21] in the context of the shell model. Effective one-body operators for the GT transition have been proposed there to account phenomenologically for core polarization, isobar and meson exchange currents, and relativistic effects, all of them theoretically evaluated by Towner and Khanna for several isotopes [22]. In these cases a single-particle model is assumed and transitions where two orbital angular momentum units are changed, known as $\ell$-forbidden transitions [23-25], are described by the introduction of a new term of the form $\left[Y^{(2)} \times \vec{\sigma}\right]^{(1)}$. In our case, IBFM-2 also considers a single nucleon coupled to a core described with interacting bosons and eventually similar higher-order corrections can be introduced, since here we are faced with transitions $3 s_{1 / 2} \rightarrow 2 d_{3 / 2}$ in the decay of $1 / 2_{1}^{+}$states in ${ }^{A} \mathrm{Cs}$ to $3 / 2_{1}^{+}$states in ${ }^{A} \mathrm{Xe}$ which cannot be accounted for properly. Instead we have estimated renormalization factors for the calculated matrix elements of the allowed transitions to obtain reasonable calculated values compared with the experimental ones. We have obtained that a renormalization factor of $1 / 3$ for the Fermi and Gamow-Teller matrix elements in the transitions $1 / 2_{1}^{+} \rightarrow 1 / 2_{1}^{+}$produces close results to the data. The dominant single-particle transition here is $3 s_{1 / 2} \rightarrow 3 s_{1 / 2}$ which is $\ell$-allowed. In contrast, for the transitions $1 / 2_{1}^{+} \rightarrow 3 / 2_{1}^{+}$a renormalization factor of 2 for the Gamow-Teller matrix elements is suitable to match the experimental data. The dominant single-particle transition in this case is $3 s_{1 / 2} \rightarrow 2 d_{3 / 2}$ which is $\ell$-forbidden. In Fig. 2 we show the $\log f t$ values affected by these renormalizations factors. It seems that for $\ell$-allowed transitions a quenching in the nuclear matrix elements, both Fermi and Gamow-Teller, is needed, while for $\ell$-forbidden transitions the Gamow-Teller nuclear matrix elements are calculated too small and need an enhancement. This could be partially due to the lack of higher-order terms in the Gamow-Teller operator, as discussed above, but probably mainly due to the limited model space. In Ref. [24] within the shell model it is shown that increasing the model space entails a reduction in the $\ell$-allowed transitions and an increase in the $\ell$-forbidden transition strengths. In any case, the number of decays studied here is small to get a strong conclusion, but we have now a tool to study thoroughly the problem of these state-dependent renormalizations, and eventually the renormalization of the axial coupling constant $g_{A}$.

\section{SUMMARY}

We have performed a free parameter calculation of the $\beta$-decay rates of ${ }^{121-131} \mathrm{Cs}$. To this end we have followed two approaches using the IBFM-2 model. In the first approach we employ the usual NOA approximation used in the microscopic boson-fermion interaction of the model and in the $\beta$-decay operators. In the second approach we do not use this approximation in the $\beta$-decay operators. When the calculated $\beta$ decay rates are compared with the corresponding experimental values, the second approach provides better global results than the first one. We have also checked that a consistent truncation in the number of states of the final Xe nuclei improves considerably the quality of the results both with and without the NOA approximation. The best results are those of the new operator obtained without using the NOA approximation and with a consistent energy cutoff between the odd-even nucleus and its even-even core. The calculated $\log f t$ values describe reasonably well the experimental systematics, although we point out that higher-order corrections to the $\beta$-decay operator may be necessary to improve them quantitatively.

In conclusion, now we have at our disposal a reliable tool to not only study single- $\beta$ decay systematically, but also to address fundamental questions related to the effective value of $g_{A}$, double- $\beta$ decay, the gallium anomaly, and/or dark matter scattering in heavy nuclei (in the last two cases the relevant nuclear matrix elements are the same as in $\beta$ decay).

Finally, it is worth noting that the IBFA-2 boson-fermion interaction currently used is based on the transfer operator obtained using NOA. Thus, a completely consistent calculation without the NOA approximation would require the recalculation of this boson-fermion interaction without using NOA. This is the final step in our program of giving a consistent and microscopically derived framework to study odd- $A$ nuclei in the context of the interacting boson-fermion model. Work along this direction is in progress and will be presented in a forthcoming publication.

\section{ACKNOWLEDGMENTS}

We thank Prof. Francesco Iachello for his helpful comments on this work. This research has been partially supported by Chilean Ministerio de Educación (Chile) under Fondecyt project 1150564, by Spanish Ministerio de Economía y Competitividad (Spain) under project FIS2014-53448-C2-1-P, and by Consejería de Economía, Innovación, Ciencia y Empleo de la Junta de Andalucía (Spain) under Group FQM-160 and project P11-FQM-7632.
[1] F. Iachello and A. Arima, The Interacting Boson Model, Cambridge Monographs on Mathematical Physics (Cambridge University, New York, 1987).
[2] F. Iachello and P. Van Isacker, The Interacting Boson-Fermion Model, Cambridge Monographs on Mathematical Physics (Cambridge University, New York, 1991). 
[3] A. Arima and F. Iachello, Ann. Phys. (NY) 99, 253 (1976); 111, 201 (1978); O. Scholten, F. Iachello, and A. Arima, ibid. 115, 325 (1978); A. Arima and F. Iachello, ibid. 123, 468 (1979).

[4] A. Arima, T. Otsuka, F. Iachello, and I. Talmi, Phys. Lett. B 66, 205 (1977); T. Otsuka, A. Arima, F. Iachello, and I. Talmi, ibid. 76, 139 (1978).

[5] F. Iachello and O. Scholten, Phys. Rev. Lett. 43, 679 (1979).

[6] C. E. Alonso, J. M. Arias, R. Bijker, and F. Iachello, Phys. Lett. B 144, 141 (1984).

[7] O. Scholten and A. E. L. Dieperink, in Interacting Bose-Fermi Systems in Nuclei, edited by F. Iachello (Plenum, New York, 1981); I. Talmi, in ibid.; O. Scholten, Ph.D. thesis, University of Groningen, 1980; Prog. Part. Nucl. Phys. 14, 189 (1985).

[8] I. Talmi, Nucl. Phys. A 172, 1 (1971).

[9] T. Otsuka and A. Arima, Phys. Lett. B 77, 1 (1978).

[10] T. Otsuka, A. Arima, and F. Iachello, Nucl. Phys. A 309, 1 (1978).

[11] F. Dellagiacoma and F. Iachello, Phys. Lett. B 218, 399 (1989); F. Dellagiacoma, Ph.D. thesis, Yale University, 1988.

[12] S. Brant, N. Yoshida, and L. Zuffi, Phys. Rev. C 70, 054301 (2004); L. Zuffi, S. Brant, and N. Yoshida, ibid. 68,
034308 (2003); N. Yoshida, L. Zuffi, and S. Brant, ibid. 66, 014306 (2002).

[13] J. Barea, C. E. Alonso, and J. M. Arias, Phys. Lett. B 737, 205 (2014).

[14] J. M. Arias, C. E. Alonso, and R. Bijker, Nucl. Phys. A 445, 333 (1985).

[15] M. P. Mendenhall et al., Phys. Rev. C 87, 032501(R) (2013).

[16] M. H. Macfarlane and J. B. French, Rev. Mod. Phys. 32, 567 (1960).

[17] A. de-Shalit and I. Talmi, Nuclear Shell Theory (Dover, New York, 2004).

[18] https://www-nds.iaea.org/livechart/.

[19] B. A. Brown and B. H. Wildenthal, Phys. Rev. C 28, 2397 (1983).

[20] B. H. Wildenthal, M. S. Curtin, and B. A. Brown, Phys. Rev. C 28, 1343 (1983).

[21] B. A. Brown and B. H. Wildenthal, Nucl. Phys. A 474, 290 (1987).

[22] I. S. Towner and F. C. Khanna, Nucl. Phys. A 399, 334 (1983).

[23] E. G. Adelberger et al., Nucl. Phys. A 417, 269 (1984).

[24] D. R. Semon et al., Phys. Rev. C 53, 96 (1996).

[25] L. Weissman et al., Phys. Rev. C 59, 2004 (1999). 